\title{
Segmentation of Skull in 3D Human MR Images Using Mathematical Morphology
}

\author{
B. Dogdas ${ }^{a}$, D. Shattuck ${ }^{b}$, and R. M. Leahy ${ }^{c}$ \\ Signal and Image Processing Institute, University of Southern California, \\ Los Angeles, CA 90089-2564
}

\begin{abstract}
We present a new technique for segmentation of skull in human T1-weighted magnetic resonance (MR) images that generates realistic models of the head for EEG and MEG source modeling. Our method performs skull segmentation using a sequence of mathematical morphological operations. Prior to the segmentation of skull, we segment the scalp and the brain from the MR image. The scalp mask allows us to quickly exclude background voxels with intensities similar to those of the skull, while the brain mask obtained from our Brain Surface Extractor algorithm ensures that the brain does not intersect our skull segmentation. We find the inner and the outer skull boundaries using thresholding and morphological closing and opening operations. We then mask the results with the scalp and brain volumes to ensure closed and nonintersecting skull boundaries. We applied our scalp and skull segmentation algorithm to several MR images and validated our method using coregistered CT-MR image data sets. We observe that our method is capable of producing scalp and skull segmentations suitable for MEG and EEG source modeling in 3D T1-weighted human MR images.
\end{abstract}

Keywords: Skull segmentation, scalp segmentation, morphology, MR image

\section{INTRODUCTION}

Magnetoencephalography (MEG) and electroencephalography (EEG) inverse problems require realistic models of the head for use in accurate computation, via boundary or finite element methods, of the mapping from neural current sources to scalp potentials and extra-cranial magnetic fields. ${ }^{1}$ Since the conductivity of skull is significantly lower than that of soft tissue, it is crucial that bone regions be included in the head model. Because of the existence of closed form solutions for the MEG and EEG forward problem, multilayer spherical models have traditionally been used to approximate the human head with a set of nested spheres representing brain, skull and scalp. Recently, representations of the head as a set of contiguous regions bounded by surface tessellations of the scalp, outer skull, inner skull, and brain boundaries have been used as a more realistic model. Using boundary element methods in conjunction with these models produces more accurate results than the multilayer spherical model ${ }^{2}$ but requires that a volumetric image of the subject's head first be segmented into its component bone and soft tissue regions.

Though CT data exhibits better bone definition than MR imagery, it does not provide good contrast in soft tissue. In addition, CT is not a preferred modality for routine anatomical imaging for segmenting structures in the head because of the X-ray exposure of the subject. Conversely, MR imaging does an excellent job of differentiating soft tissues, including cerebral cortex and blood vessels, but it does not provide accurate detail of bone structures, such as the skull, because of the weak MR signals produced in bone. Segmentation of skull from MR images therefore presents a challenging problem. However, methods for segmenting skull from MR images will make it possible to obtain individual models of scalp, skull, and brain from a single acquisition of a complete head at high resolution.

Send correspondence to R. M. Leahy

a: E-mail: dogdas@sipi.usc.edu, Telephone: 12137406430

b: E-mail: shattuck@sipi.usc.edu, Telephone: 1213740 4143,

c: E-mail: leahy@sipi.usc.edu, Telephone: 1213740 4659,

Address: 3740 McClintock Ave, University of Southern California, Los Angeles, CA 90089-2564, USA 


\section{RELATED WORK}

Though numerous MR image segmentation techniques are described in the literature, there is little research dedicated to the problem of segmentation of skull in MR images, as CT is typically used for this purpose. Held et al. used a Markov random field (MRF) approach to classify MR images into gray matter, white matter, CSF, scalp-bone and background. ${ }^{3}$ Heinonen et al. used thresholding and region growing to segment bone in MR image volumes. ${ }^{4}$ In other research, Rifai et al. applied a deformable model to segment skull from MR images. $^{5}$ Chu and Takaya detected the skin-skull and skull-brain boundaries using thresholding and Laplacian of Gaussian (LoG) operations on successive transverse slices. ${ }^{6}$ Soltanian-Zadeh and Windham applied a multiscale approach where they made use of both CT and MR image information. ${ }^{7}$ Studholme et al. ${ }^{8}$ also used CT information for segmentation of skull in MR images of the same subject.

The method in Held et al. does not guarantee continuous bounding contours and was not intended for segmentation of the skull in MR images. With thresholding and region growing methods, the segmentation of certain bone regions, such as ocular globes, is difficult because of partial volume effects. Deformable models can be attracted to incorrect boundaries resulting in the potential inclusion of skin, muscles, eyes, and inner ear in the segmented skull. Performing the segmentation on individual slices does not allow the method to exploit the connected 3D structure of the skull. The choice of a multi-modal approach (CT/MR), while attractive in providing accurate detail of both skull and soft tissue, is not generally practical since acquisition using both modalities is rarely performed either in volunteer or clinical studies. Therefore there is still a need for a robust method for finding skull boundaries in MR. Here we describe our approach to this problem which we base on a sequence of morphological operations.

\section{OBTAINING ANATOMICAL SURFACES WITH MORPHOLOGICAL PROCESSING}

Binary mathematical morphology is an algebraic system based on set theory that provides two basic operations: dilation and erosion. Combinations of these operations enable underlying object shapes to be identified and reconstructed from their noisy distorted forms. A combination of dilation and erosion gives rise to two additional operations, opening and closing, which form the basis for most morphological processing. ${ }^{9}$ Morphological approaches have already been widely used in segmentation of MR imagery, ${ }^{10},{ }^{11},{ }^{12}$.

Accurate segmentation of skull in MR is difficult because the MR signal from bone is weak and often indistinguishable from air, and because of the complex topology of the skull. Certain parts of the skull such as the ocular globes and portions of the upper region of the skull are thin compared to the resolution currently available in MR images. This problem is confounded by partial volume effects that tend to reduce the apparent thickness of the skull. In regions in which bone and air are separated by thin layers of tissue (e.g., sinuses, external auditory canals) it is often difficult to differentiate between air and bone voxels. ${ }^{5}$

Our method performs skull and scalp segmentation using a sequence of mathematical morphological operations. Prior to the segmentation of skull, we segment the brain and scalp from the MR image. We exclude the brain from the image volume to obtain an estimate of the intensity distribution of the scalp and the skull regions. We also use the brain region to ensure that our skull boundary does not intersect the brain boundary. We use the scalp segmentation to distinguish between dark voxels that may be skull or air, since these areas will have overlapping intensity distributions.

\subsection{Mathematical Morphology}

Morphological operators use a binary image and a structuring element as input and combine them using intersection, union, inclusion or complement operators. We use the following structuring elements in our algorithm: cube, 3D cross, and 3D octagon. The 3D octagon, which we denote by $\mathrm{O}_{2}$, is an approximation of a sphere and is defined as the dilation of a $3 \mathrm{D}$ cross by a $3 \mathrm{D}$ cube. This is analogous to an octagon shape in $2 \mathrm{D}$, which is produced by the dilation of a $3 \times 3$ square by a $3 \times 3$ cross. Figure 1 shows the basic structuring elements we use in our algorithm and their corresponding $2 \mathrm{D}$ structuring elements. 


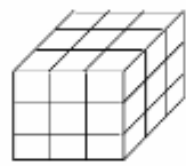

Cube $\left(C_{1}\right)$

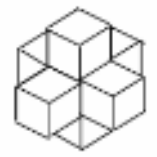

3D $\operatorname{Cross}\left(R_{1}\right)$

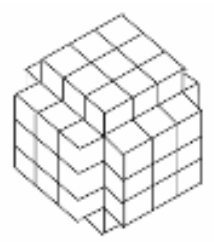

$3 \mathrm{D} \operatorname{Octagon}\left(\mathrm{O}_{2}\right)$

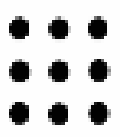

Square

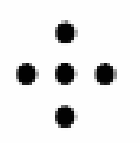

Cross

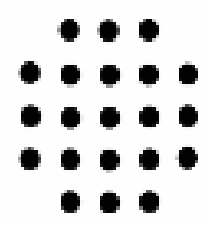

Octagon

Figure 1. Basic structuring elements used in our algorithm: $\operatorname{Cube}\left(C_{1}\right), 3 \mathrm{D} \operatorname{Cross}\left(R_{1}\right), 3 \mathrm{D} \operatorname{Octagon}\left(O_{2}\right)$ and their corresponding 2D structuring elements: Square, Cross and Octagon.

Dilation and erosion by larger structuring elements is performed by applying the corresponding structuring elements multiple times. For instance if we want to apply a dilation operation by a cubic structuring element of radius 4 , we apply dilation by a cube 3 times:

$$
C_{4}=C_{1} \oplus C_{1} \oplus C_{1} \oplus C_{1}
$$

We use the following mathematical symbols for the morphological operators in the description of our algorithm: $\oplus$ for dilation, $\ominus$ for erosion, o for opening, • for closing, and $\odot$ for a special form of closing operation with hole filling, described in detail below.

\subsection{Segmentation of Brain}

We segment the brain using the Brain Surface Extractor (BSE) software, v.3.0. ${ }^{13}$ BSE identifies the brain using anisotropic diffusion filtering, Marr-Hildreth edge detection, and morphological operations. Anisotropic diffusion filtering improves the edge definitions in the MR image by smoothing non-essential gradients in the volume without blurring steep edges. The Marr-Hildreth edge detector identifies important anatomical boundaries such as the brain/skull boundary. The brain is identified and refined using a sequence of morphological and connected component operations. ${ }^{10}$

\subsection{Segmentation of Scalp}

The scalp or skin surface is the interface between background and the head. To segment the scalp, we first apply simple thresholding to identify the boundary between the skin and surrounding air. This transition is quite sharp, so our primary concern is in finding an appropriate threshold to remove noise in the background. After we extract the brain from the MR image, we are left with low intensity regions (the background and skull) and higher intensity regions (fat, skin, muscle and other soft tissues). We compute an empirical skull threshold as the mean of the histogram after extraction of the brain, and we estimate the scalp threshold as the mean of the histogram above the skull threshold. These thresholds give the algorithm an initial estimate of the range of the skull and scalp intensities. They may be adjusted by the user to improve the segmentation.

Thresholding produces a set of foreground voxels described as

$$
X_{t}=\left\{k \in V: x_{k} \geq t_{\text {scalp }}\right\}
$$

where $t_{\text {scalp }}$ is the scalp threshold, $\mathrm{V}$ is the entire MR volume, and $x_{k}$ is the $k^{t h}$ measurement value.

After thresholding, the volume will still contain background voxels due to noise. It will also have cavities in the head due to regions of tissue that have low intensity values. These cavities will often be connected to the background through the sinuses or auditory canals. To address these problems, we apply a modified morphological closing operation. A traditional closing consists of a dilation followed by an erosion using a structuring element S. Our closing operation, which we denote by $\odot$, performs a hole filling operation between dilation and erosion. This will fill any cavities that are disjoint from the background after dilation. We obtain 
the scalp volume by applying our modified closing operation to $X_{t}$, the thresholded volume, using a structuring element $\mathrm{O}_{2}$ :

$$
X_{i}=X_{t} \odot O_{2} .
$$

Provided that the dilation closes the auditory canal and sinuses, this hole-filling closing operation will have the effect of forming a single volume that contains the entire head, including brain and skull as well as scalp. We select the largest foreground connected component as this head volume,

$$
X_{l c}=S C C\left(X_{i}\right),
$$

where $S C C$ selects the largest foreground connected component.

We remove the remaining noise by applying an opening operation with a cube of size 2 to the resultant volume,

$$
X_{\text {scalp }}=X_{l c} \circ C_{2} .
$$

\subsection{Segmentation of Skull}

Finding the skull in MR images is difficult because the skull appears as a set of dark voxels in T1 weighted MR and typically has a width of only three or four voxels. To find the skull volume, we have to identify both the outer and inner skull boundary.

\subsubsection{Segmentation of Outer Skull}

To find the outer skull, we begin by labeling the dark voxels in the image with a thresholding operation. We estimate a threshold for skull as the mean of the histogram of the non-zero voxels in the MR volume that are not identified as brain,

$$
X_{\text {dark }}=\left\{k \in V: x_{k} \leq t_{\text {skull }}\right\},
$$

where $t_{\text {skull }}$ is the skull threshold, $\mathrm{V}$ is the entire MR volume, and $x_{k}$ is the $k^{\text {th }}$ measurement value.

Some regions inside the head will not be included in the skull volume after thresholding. To fill these regions, we take the union of our thresholded image, $X_{d a r k}$, with a dilated brain mask. The dilated brain mask is obtained by dilating the mask from BSE with a $3 \times 3$ cube of radius 2, denoted as $C_{2}$.

$$
\begin{aligned}
B_{d m} & =B_{\text {mask }} \oplus C_{2} \\
X_{u} & =X_{\text {dark }} \cup B_{d m} .
\end{aligned}
$$

Next, we take the intersection of this volume with a modified scalp volume to include only the dark voxels that lie within the head. We obtain this modified scalp volume by applying an opening operation with structuring element a cube of size 12, followed by an erosion operation with a cube of size 1 as the structuring element. These two operations generate a modified scalp volume that typically does not include the ears or nose. In particular, this volume excludes portions of the ear canal and nasal sinuses whose intensity values are indistinguishable from skull. By masking the skull with this modifed scalp volume, we ensure that these regions do not appear incorrectly as part of the skull, and also that the skull does not intersect the scalp boundary.

$$
\begin{aligned}
X_{e s} & =\left(X_{\text {scalp }} \circ C_{12}\right) \ominus C_{1} \\
X_{m} & =X_{u} \cap X_{e s} .
\end{aligned}
$$

The largest connected region resulting from this operation will be the closed volume bounded by the outer skull, but $X_{m}$ may contain additional connected components such as the eyeballs. Thus we now select the largest connected component in $X_{m}$ :

$$
X_{l c}=S C C\left(X_{m}\right) .
$$


We then close the boundary of the outer skull using a closing operation with the structuring element $O_{4}$,

$$
X_{c}=X_{l c} \bullet O_{4} .
$$

This closing operation may cause the volume to intersect the scalp, so our final operation is to again mask the volume with the eroded scalp to enforce the physical constraint that the outer skull boundary lies within the scalp.

$$
X_{\text {out skull }}=X_{c} \cap X_{\text {es }} .
$$

The resulting volume $X_{\text {out skull }}$ is a closed volume bounded by the outer skull boundary.

\subsubsection{Segmentation of Inner Skull}

Finding the inner skull is more difficult than finding the scalp and outer skull. We have to be careful to include all parts of the brain and the CSF within the inner skull boundary. In some MR volumes with low SNR, skull is difficult to distinguish from CSF and therefore CSF may be misinterpreted as skull. In order to overcome this problem we use the outer skull and brain masks to restrict the allowed locations of the inner skull boundary.

First we mask the MR image with a skull mask that we obtain from the outer skull volume by erosion with the structuring element $C_{1}$.

$$
\begin{aligned}
X_{s m} & =X_{\text {out skull }} \ominus C_{1} \\
X_{o} & =V \cap X_{\text {sm }} .
\end{aligned}
$$

This ensures that the resultant inner skull will lie inside the outer skull. Then, using the same threshold as for the outer skull, we remove the skull from this volume by thresholding,

$$
X_{\text {inner }}=\left\{k \in X_{o}: x_{k} \geq t_{\text {skull }}\right\} .
$$

The resulting volume should represent the region bounded by the inner skull boundary. It appears noisy due to various remaining confounds such as bright voxels due to diploic fat within the skull. We can remove these regions by applying an opening operation. However, the thresholding operation also produces holes in the volume resulting from CSF voxels with intensities similar to that of the skull. Therefore, before applying the opening operation we first take the union of the inner skull with a dilated brain mask to directly fill these non-skull regions. We use a structuring element of $C_{1}$ for dilating the brain. With this operation we ensure that the inner skull encloses the brain mask. Next, we perform the opening operation to remove dipolic fat and other extraneous materials using a structuring element of $O_{4}$,

$$
\begin{aligned}
B_{d m} & =B_{\text {mask }} \oplus C_{1} \\
X_{u} & =X_{\text {inner }} \cup B_{d m} \\
X_{\text {open }} & =X_{u} \circ O_{4} .
\end{aligned}
$$

While the previous steps will remove most CSF regions from the estimated skull mask, there may be regions where the dilated brain mask alone does not encompass all CSF, which therefore appears as part of the skull. To overcome this problem we impose a physical constraint on skull thickness in our algorithm and make sure that the skull volume does not exceed $4 \mathrm{~mm}$. We apply an erosion operation to the outer skull volume with a structuring element of $\mathrm{O}_{4}$ and obtain the inner skull after masking the volume with this eroded skull,

$$
\begin{aligned}
X_{\text {es }} & =\left(X_{\text {outer skull }} \ominus O_{4}\right) \\
X_{\text {inner skull }} & =X_{\text {open }} \cap X_{\text {es }} .
\end{aligned}
$$

While this operation will lead to underestimation of skull thickness in subjects with thicker skulls, it is a practical solution to the problem that CSF and skull are often indistinguishable. This thickness constraint can also be modified by the user. 


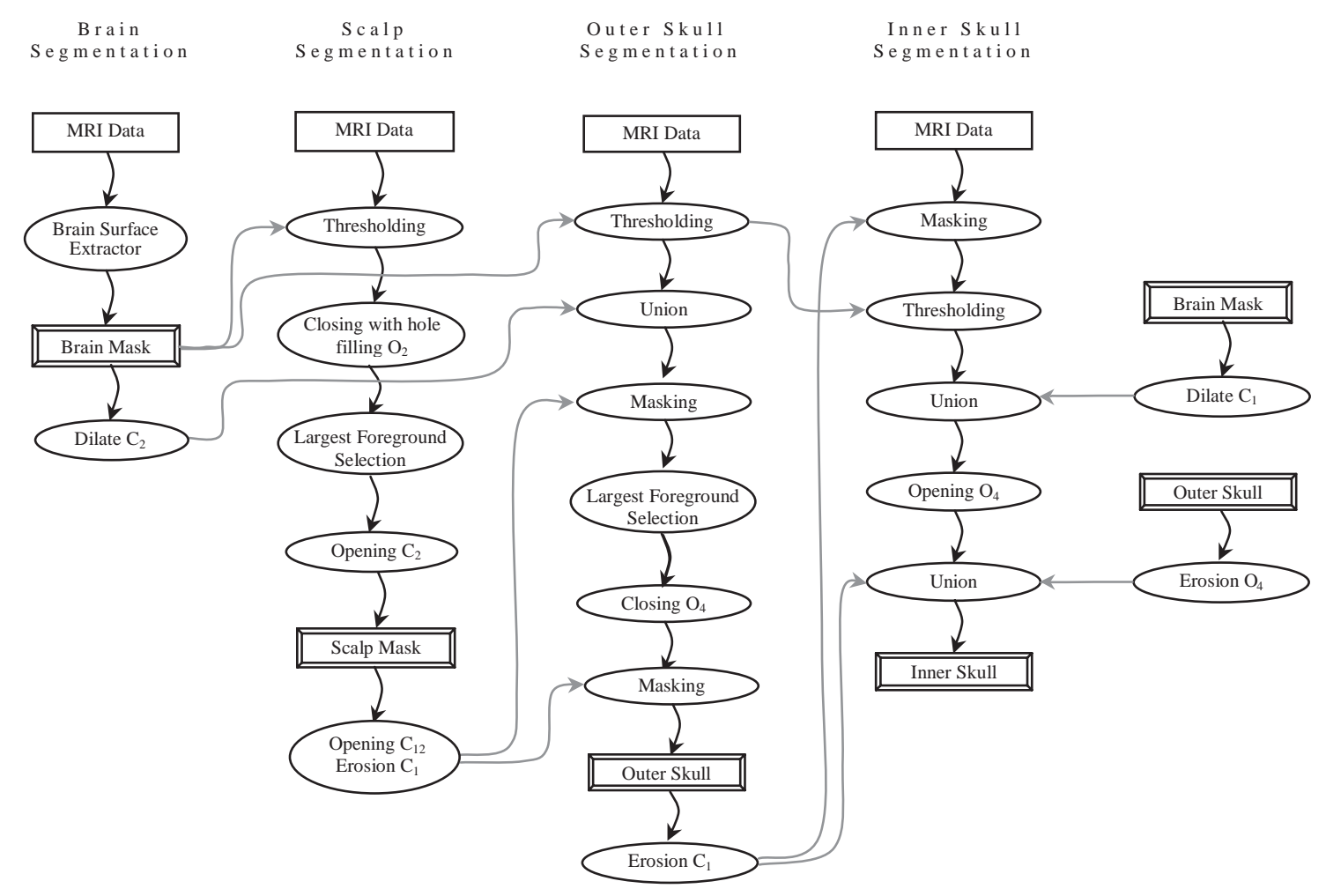

Figure 2. Block diagram of our algorithm for scalp and skull segmentation using morphological operations. On completion, we apply additional masking operations to ensure that scalp, outer skull, inner skull and brain masks do not intersect each other.

Because of the partial volume effects and the resolution of the MR data, the skull can be very thin in some parts of the image. This may cause problems in our segmentations because the resultant surface tessellations of the scalp, outer skull, inner skull and brain boundaries may intersect. We need to constrain our segmentation results to make sure that each structure does not intersect with the neighboring surfaces. We apply this constraint from inside to outside. First we dilate the brain mask with structuring element $C_{1}$ and take the union of this mask with the inner skull. We repeat the same procedure for the outer skull and scalp. We obtain the outer skull by taking its union with the dilated inner skull; we obtain the scalp by taking its union with the outer skull dilated with structuring element $C_{1}$. This correction procedure guarantees that we obtain nonintersecting boundaries. Figure 2 describes a complete block diagram of our algorithm for scalp and skull segmentations using morphological operations.

\section{RESULTS}

We implemented our scalp and skull algorithm using the $\mathrm{C}++$ programming language. We applied our scalp and skull segmentation algorithm to more than $40 \mathrm{MR}$ images and validated it using 8 coregistered CT-MR data sets. The CT-MR data sets were provided as part of the project, "Retrospective Image Registration Evaluation". ${ }^{14}$ In this database, the T1-weighted MR data were obtained using a Magnetization Prepared RApid Gradient Echo (MP-RAGE) sequence. This is a rapid gradient-echo technique in which a preparation pulse (or pulses) is applied before the acquisition sequence to enhance contrast. ${ }^{15}$ The dimensions of the MR data were $256 \times 256 \times 128$ with resolution on the average of $0.98 \mathrm{~mm} \times 0.99 \mathrm{~mm} \times 1.484 \mathrm{~mm}$. The corresponding CT scans had $3 \mathrm{~mm}$ slice thickness with slice dimensions $512 \times 512$, with resolution on the average of $0.419 \mathrm{~mm}$ x $0.419 \mathrm{~mm}$. The number of slices for each volume varied between 42 and 49 . 


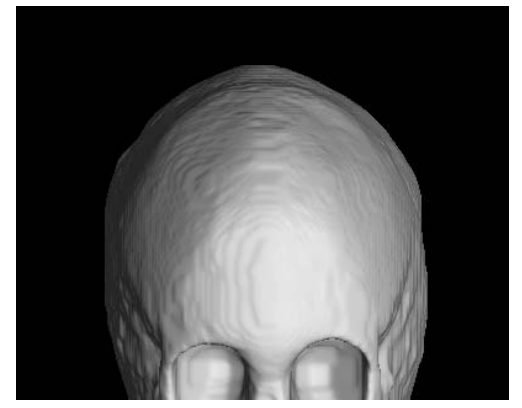

outer skull

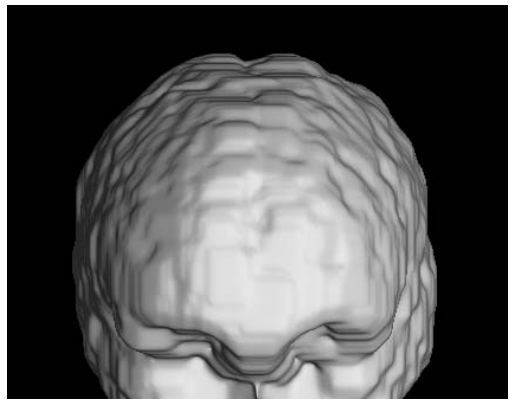

inner skull

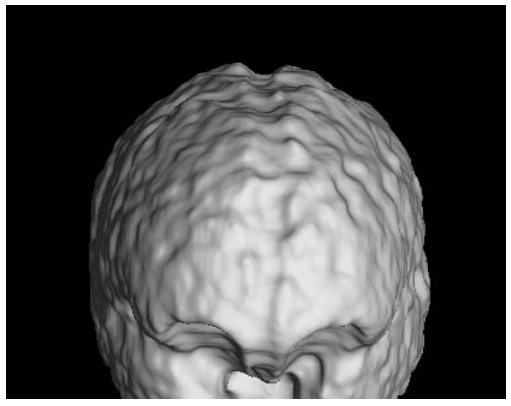

brain

Figure 3: Surface tesselations of the outer skull, inner skull, and brain obtained from one of the MR data sets

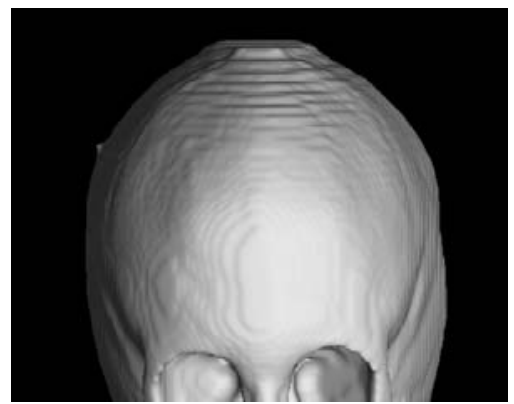

outer skull

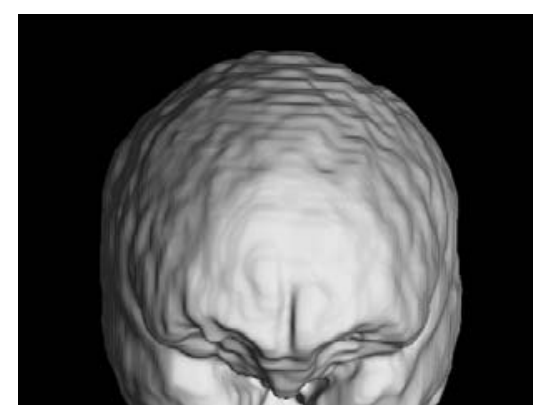

inner skull

Figure 4: Surface tesselations of the outer skull and inner skull of the corresponding CT data set.

We processed the $\mathrm{CT}$ and MR volumes by registering each CT volume to its corresponding MR image. We registered these data using the 3D Multi-Modal Image Registration Viewer Software (RView 8.0w Beta). ${ }^{16}$ After aligning the volumes, we labeled the skull in the CT data sets using thresholding. We used closing and flood filling procedures to fill the diploic spaces within the skull. Since bone in CT is easily distinguished from other tissues in the head, this simple operation produced accurate results compared to those we expect from MR and we treated these images as a gold standard against which to compare the MR segmentations.

Figure 3 shows the results of our surface finding method as tessellations of the outer skull, inner skull and brain for one of the MR data sets. Figure 4 shows the corresponding surface tessellations of the outer skull and inner skull computed from the corresponding coregistered CT data set. Surface tesselations in all cases were computed using the Marching Cubes algorithm ${ }^{17}$ and rendered using our BrainSuite software. ${ }^{10}$

To assess the performance of our algorithm we computed the Dice coefficients between regions obtained from the CT and MR images. This metric measures the similarity of two sets and ranges from 0 for sets that are disjoint to 1 for sets that are identical. This index is a special case of the kappa index that is used for comparing set similarity. ${ }^{18}$ The Dice coefficient is defined as:

$$
k\left(S_{1}, S_{2}\right)=\frac{2\left|S_{1} \cap S_{2}\right|}{\left|S_{1}\right|+\left|S_{2}\right|}
$$

Skull morphology in the lower portion of the head is extremely complex and performance of our algorithm in these regions is unreliable. However, forward modeling calculations in MEG and EEG are primarily affected by the scalp and the skull boundaries in the regions above the plane passing through the nasion and inion and perpendicular to the sagittal planes. Consequently, we restricted our evaluation to the skull volume above this 


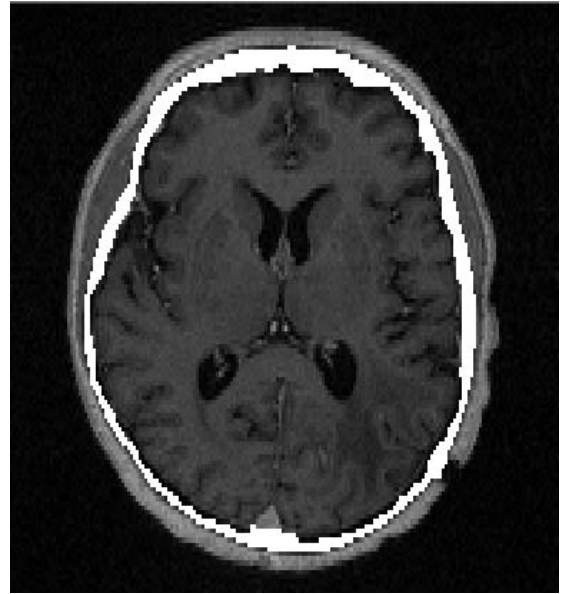

(a) Overlay of the MR skull onto MR

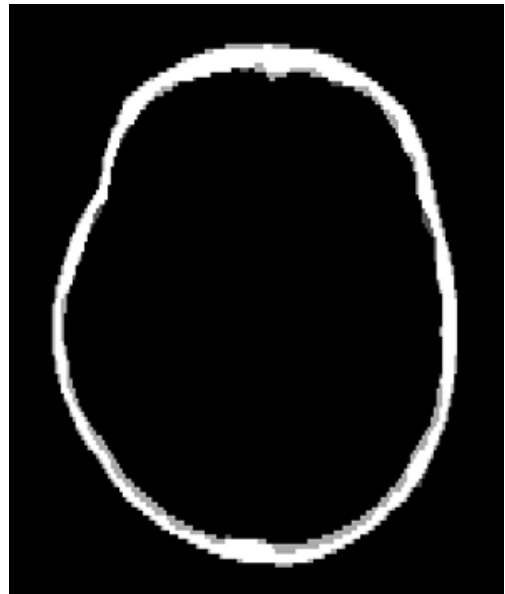

(b) Overlay of MR and CT skulls

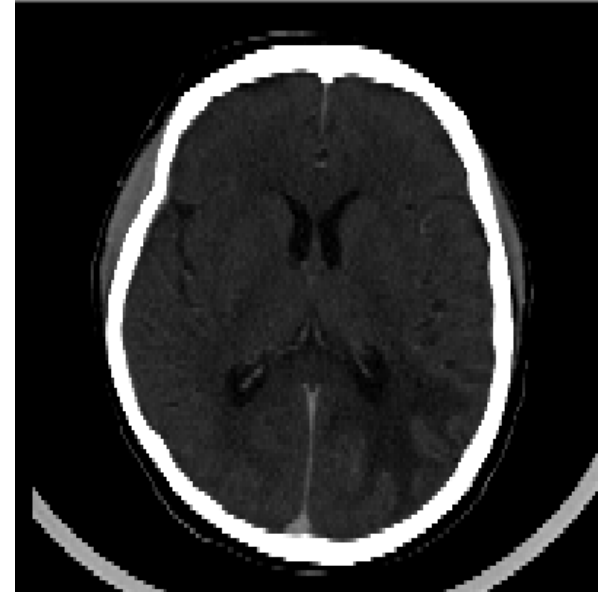

(c) Overlay of CT skull onto CT

Figure 5. Overlay of the MR and CT skulls on a transaxial slice of one of the MR images and its corresponding coregistered CT: (a) overlay of the MR skull on MR: white region corresponds to skull; (b) overlay of MR and CT skulls; white: CT and MR skulls agree, dark gray: MR skull only, light gray: CT skull only, black: background; (c) overlay of the CT skull on coregistered CT.

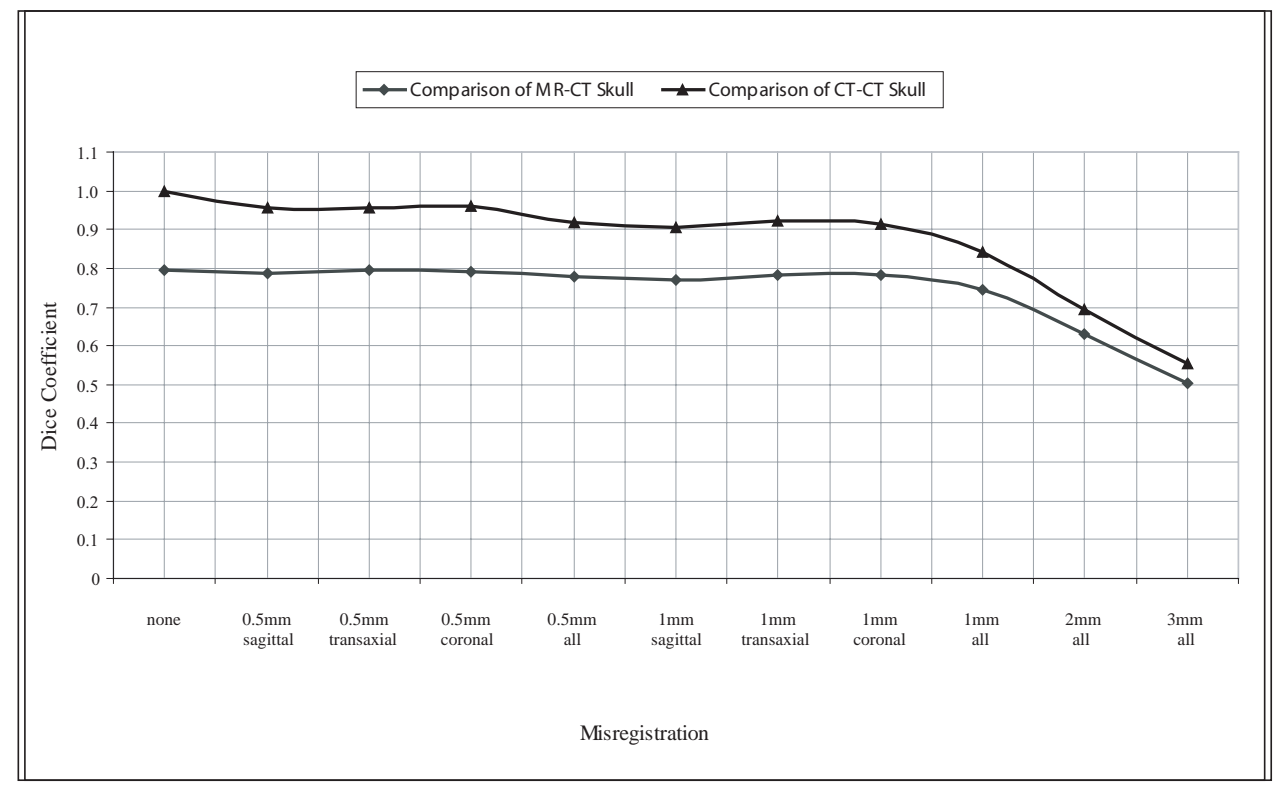

Figure 6. Plot of the Dice coefficients for the comparison of $0.5 \mathrm{~mm}, 1 \mathrm{~mm}, 2 \mathrm{~mm}$ and $3 \mathrm{~mm}$ misregistered CT skull with itself and MR skull in the direction of sagittal, transaxial and coronal sections. $0.5 \mathrm{~mm}$ in all directions $=0.8660 \mathrm{~mm}$ misregistration, $1 \mathrm{~mm}$ in all directions $=1.7321 \mathrm{~mm}$ misregistration. 


\begin{tabular}{|c|c|}
\hline & Skull \\
\hline Patient1 & 0.7608 \\
Patient2 & 0.7134 \\
Patient3 & 0.6364 \\
Patient4 & 0.7940 \\
Patient5 & 0.7852 \\
Patient6 & 0.7912 \\
Patient7 & 0.7254 \\
Patient8 & 0.7964 \\
\hline Average & $0.7504 \pm 0.1140$ \\
\hline
\end{tabular}

Table 1. Dice coefficients comparing skull volumes (i.e., the region between inner and outer skull boundaries) for eight coregistered CT and MR image data sets.

plane. We compared each skull (the region between the outer and the inner skull boundaries) obtained from the MR image with that extracted from the corresponding coregistered CT. Table 1 lists the Dice coefficients for the $8 \mathrm{MR} / \mathrm{CT}$ data sets used in this study. Figure 5 shows an overlay of the MR and CT skulls on transaxial $\mathrm{MR}$ and $\mathrm{CT}$ sections for one of the data sets.

To assess the implications of the results in Table 1, we compared these values with the Dice coefficients computed from misregistered copies of the $\mathrm{CT}$ skull for one of the CT/MR data sets. In this way we can compute the degree of misregistration between a CT image and the head which would account for a similar level of error as that between our CT gold standard and the MR skull. In MEG and EEG applications, spatial localization errors between brain images and the sensor locations on the order of $2 \mathrm{~mm}$ is common. We therefore anticipate that errors in the skull volume equivalent to misregistration of 1-2mm would be acceptable.

We shifted the CT skull volume by $0.5 \mathrm{~mm}$ and $1 \mathrm{~mm}$ separately along each of the three cardinal axes in turn. We also shifted the skull volume by $1 \mathrm{~mm}, 2 \mathrm{~mm}$ and $3 \mathrm{~mm}$ along each of the three axes to create an additional three misregistered skull volumes. These latter three volumes correspond to shifts by $\sqrt{3}, 2 \sqrt{3}$ and $3 \sqrt{3} \mathrm{~mm}$. We then computed the Dice coefficients between these shifted and original CT skulls. We also performed the same shifts on the computed MR skulls to determine the robustness of the MR extraction method to misregistration. The Dice coefficients for each of these comparisons is plotted in Figure 6. The figure indicates that the Dice coefficient for the MR/CT skull comparison is equivalent to that of a $\sqrt{3} \mathrm{~mm}$ misregistration between the $\mathrm{CT}$ and the subject's true head location.

\section{DISCUSSION AND CONCLUSIONS}

We applied our scalp and skull segmentation algorithm to eight CT/MR coregistered data sets. To assess the performance of our algorithm we computed the Dice coefficients, which give a measure of the similarity of the segmentation results we obtained from $\mathrm{CT}$ and MR. We performed these comparisons on the upper portion of the head by defining a plane that passes through the nasion and the inion and obtained a Dice coefficient of $0.7504 \pm 0.1140$ for the skull comparisons. Comparison of these results with the effect of shifting the CT skull with respect to itself reveals that these results are comparable to misregisration on the order of $1.7 \mathrm{~mm}$ between an accurately determined CT-based skull and the subject's head. We can therefore conclude that our algorithm generates scalp and skull volumes that may be of acceptable accuracy for MEG and EEG source modeling since registration errors on the order of $1-2 \mathrm{~mm}$ are typical for these modalities.

In some instances one may need to adjust the scalp and skull thresholds to obtain reasonable estimates of the skull volumes. However, the thresholds can be quickly adjusted and identification of the scalp and skull surfaces on a 256-256-128 volume using the method described here requires less than 20 seconds of processing time on a $1 \mathrm{GHz}$ Pentium III processor. 


\section{ACKNOWLEDGMENTS}

The images were provided as part of the project, "Retrospective Image Registration Evaluation", National Institutes of Health, Project Number 1 R01 CA89323, Principal Investigator, J. Michael Fitzpatrick, Vanderbilt University, Nashville, TN. We thank Dr. Colin Studholme for providing 3D Multi-model Image Registration Viewer (RView) software $8.0 \mathrm{w}$ Beta. ${ }^{16}$ This work was supported in part by the National Institute of Mental Health Grant RO1-MH53213.

\section{REFERENCES}

1. J. C. Mosher, R. M. Leahy, and P. Lewis, "EEG and MEG: forward solutions for inverse methods," IEEE Transactions on Biomedical Engineering 46 3, pp. 245-259, March 1999.

2. J. J. Ermer, J. Mosher, S. Baillet, and R. M. Leahy, "Rapidly recomputable EEG forward models for realistic head shapes," Phys Med Biol 46 4, pp. 1265-1281, Apr 2001.

3. K. Held, E. Kops, B. Krause, W. Wells, R. Kikinis, and H.W. Muller-Gartner, "Markov random field segmentation of brain MR images," IEEE Transactions on Medical Imaging 16, pp. 878-886, Dec 1997.

4. T. Heinonen, H. Eskola, P. Dastidar, P. Laarne, and J. Malmivuo, "Segmentation of T1 MR scans for reconstruction of resistive head models," Computer Methods and Programs in Biomedicine 54, pp. 173181, 1997.

5. H. Rifai, I. Bloch, S. Hutchinson, J. Wiart, and L. Garnero, "Segmentation of the skull in MRI volumes using deformable model and taking the partial volume effect into account," SPIE Proceedings 3661, pp. 288-299, 1999.

6. C. Chu and K. Takaya, "3-Dimensional rendering of MR images," WESCANEX 93. Communications, Computers and Power in the Modern Environment Conference Proceedings, IEEE, pp. 165-170, 1993.

7. H. Soltanian-Zadeh and J. Windham, "A multi-resolution approach for intracranial volume segmentation from brain images," Medical Physics 24 12, pp. 1844-1853, Dec 1997.

8. C. Studholme, D. L. G. Hill, and D. Hawkes, "Automated 3D registration of MR and CT images of the head," Medical Image Analysis 1 2, pp. 163-175, 1996.

9. D. Nadadur and R. M. Haralick, "Recursive binary dilation and erosion using digital line structuring elements in arbitrary orientations," IEEE Transactions on Image Processing 9, pp. 749-759, May 2000.

10. D. Shattuck and R. M. Leahy, "Brainsuite: An automated cortical surface identification tool," Medical Image Analysis in press, 2001b.

11. M. Bomans, K. Hohne, U. Tiene, and M. Riemer, "3D segmentation of MR images of the head for 3D display," IEEE Transactions on Medical Imaging 9, pp. 177-183, June 1990.

12. M. E. Brummer, R. M. Merseau, R. L. Eisner, and R. J. Lewine, "Automatic detection of brain contours in MRI data sets," IEEE Transactions on Medical Imaging 12, pp. 153-166, 1993.

13. S. Sandor and R. M. Leahy, "Surface-based labeling of cortical anatomy using a deformable database," IEEE TMI 16, pp. 41-54, 1997.

14. J. West, J. M. Fitzpatrick, M. Y. Wang, and B. M. Dawant, et al., "Comparison and evaluation of retrospective intermodality image registration techniques," Journal of Computer Assisted Tomography 21, pp. 554-566, 1997.

15. J. P. Mugler and J. R. Brookeman, "Three-dimensional magnetization-prepared rapid gradient-echo imaging (3D MP RAGE)," Magnetic Resonance in Medicine 15, pp. 152 - 157, 1990.

16. C. Studholme, D.L.G. Hill, and D.J. Hawkes, "An overlap invariant entropy measure of 3D medical image alignment," Pattern Recognition 32 1, pp. 71-86, Jan 1999.

17. W. Lorensen and E. Harvey, "Marching cubes: A high resolution 3D surface construction algorithm," ACM Computer Graphics 21 24, pp. 163-169, 1987.

18. A. P. Zijdenbos, B. M. Dawant, R. A. Margolin, and A. Palmer, "Morphometric analysis of white matter lesions in MR images," IEEE Transactions on Medical Imaging 13, pp. 716-724, Dec 1994. 